\title{
LÓGICA DEL DESCUBRIMIENTO Y LÓGICA DE LA JUSTIFICACIÓN: ¿OPOSICIÓN O COMPLEMENTARIEDAD?
}

\author{
Javier Kasahara B.*
}

\begin{abstract}
En este artículo se aborda la distinción contemporánea en filosofía de la ciencia entre una lógica del descubrimiento y una lógica de la investigación. Tal distinción opone el descubrimiento como acción creadora a la actividad científica. A partir de la distinción entre lógica y razonamiento, se sugiere que es plausible recuperar el descubrimiento como parte de la actividad científica, contrario a planteamientos como los de Popper.
\end{abstract}

Palabras claves: lógica, descubrimiento, justificación, Popper.

\section{LOGIC OF THE DISCOVERY AND LOGIC OF THE JUSTIFICATION: OPPOSITION OR COMPLEMENTARITY?}

This paper explains in the philosophy of science the contemporary distinction between logic of discovery and logic of research. That distinction opposes the discovery as a creative action to the scientific activity. From the distinction between logic and reasoning, the paper suggests that it seems reasonable to recover discovery as part of the scientific activity, in opposition to Popper's approaches.

Keywords: logic, discovery, justification, Popper.

* Universidad Católica de la Santísima Concepción, Concepción, Chile. Correo electrónico: jkasahara@ucsc.cl 


\section{Lógica del descubrimiento y Lógica de la investigación científica: Antecedentes}

LA Distinción REALIZADA POR POPPER ENTRE UnA "lógica de la investigación científica" y una "lógica del descubrimiento" sintetiza en gran medida el marco dentro del cual se han dado las principales reflexiones contemporáneas en torno al valor de las teorías científi$\operatorname{cas}^{2}$. En términos más precisos, una LDD tiene que ver con el "origen, creación, génesis e invención de teorías e hipótesis", mientras que una LIC se relaciona con la "evaluación, testeo, defensa, éxito, verdad y confirmación"3.

Popper, por ejemplo, es taxativo al indicar que: “[...] mi opinión del asunto -valga lo que valiere- es que no existe, en absoluto, un método lógico de tener nuevas ideas, ni una reconstrucción lógica de este proceso"'s. En parte dicha distinción y por extensión, oposición, entre el ámbito del descubrimiento y el de la justificación viene ya sugerido en los intentos por superar el psicologismo que muchos filósofos contemporáneos denunciaron a fines del siglo XIX y comienzos del siglo XX. En este sentido, era esperable que el descubrimiento fuese ligado al ámbito de la subjetividad o de las intuiciones creadoras, siguiendo el decir de Bergson ${ }^{5}$.

Esta distinción tiene su complemento en el ya clásico texto de Reichenbach Experiencia y predicción de 1938, donde distingue entre un contexto de descubrimiento y un contexto de justificación. Con ello se configura, en gran medida, el panorama contemporáneo al interior de la filosofía de la ciencia sobre esta cuestión, estableciéndose dos dimensiones distintas y contrapuestas. El contexto de la justificación correspondería a los elementos propios que permiten validar una teoría científica como tal, mientras que el contexto de

1 De aquí en adelante emplearemos las abreviaciones LIC y LDD respectivamente.

2 Con ello nos referimos a un sentido epistémico y no ético. Para la distinción que alude a Popper, el problema de fondo es determinar en el caso específico de las ciencias empíricas un criterio para validar un conjunto de proposiciones (sistemas de enunciados o sistemas de teorías) como conocimiento científico, lo que él mismo denomina el criterio de demarcación. Cfr. Popper, K., La lógica de la investigación científica, Tecnos, Madrid, 1980, p. 27 y ss.

3 Kordig, C.R., "Discovery and Justification", Philosophy of Science, Vol. 45, 1978, p. 110.

4 POPPER, K., op. cit., p. 31.

5 Ibid. 
descubrimiento se refiere a todo lo relativo a la formulación de una hipótesis, concretamente al acto creador que llevó a un determinado científico a postular una hipótesis ante un problema. Lo central para este planteamiento, como remarca Popper, es el carácter irracional del descubrimiento, en contraposición a la justificación, donde se requiere de una contrastación, esto es, un conjunto de reglas o pasos lógicamente establecidos que permiten validar la teoría ${ }^{6}$.

Sobre lo anterior es posible determinar la ruta que ha seguido, en gran medida, la filosofía de la ciencia durante el siglo XX, esto es, la inviabilidad de asumir como parte de un programa investigativo en ciencia una lógica del descubrimiento. Así, la generación de hipótesis queda disociada de su posterior contrastación, pues sería parte de un contexto distinto al de la ciencia, poseyendo un interés tan solo tangencial, debido a que resulta imposible determinar una demostración de su generación ${ }^{7}$. En gran medida esta distinción -que encontramos en autores como Reichenbach o Popper- obedece a la especialización que surge en ciencia y a las problemáticas internas que adolece la filosofía de la ciencia a comienzos del siglo XX, por ejemplo con el positivismo y que a la base encuentra un denominador común con en el problema del psicologismo. Esto se tradujo en una reformulación de las teorías del método modernas de los siglos XVII y XVIII principalmente, donde no había una necesidad de articular lo que contemporáneamente entendemos como filosofía de la ciencia. En efecto, para un Descartes o un Leibniz, la formulación de una idea a modo de hipótesis conllevaba eo ipso su justificación, de ahí la importancia del método como reglas o pasos a seguir. Esto es lo que se ha denominado como una identidad entre ambas, vale decir el método correcto del descubrimiento = método correcto de justificación. Esta identidad implicó un importante respaldo al proyecto moderno en ciencias, dada la unidad metodológica de la ciencia que implicaba, estableciéndose con ello que descubrimiento y justificación serían el anverso y reverso de un mismo tema ${ }^{8}$.

Con todo, lo cierto es que el interés al interior de la filosofía de la ciencia por la actividad creadora sigue presente y nos interpela a cuestionar si la barrera entre creación y demostración es tan nítida como muchos teóricos de la ciencia, como Popper, han pretendido. El propio Einstein tenía presente que la búsqueda de las leyes universales estaban fuera de una lógica, y por lo mismo requerían de una suerte de intuición -Einfühlung-al momento de enfrentarse con los objetos del mundo.

No obstante esta oposición predominante en el discurso contemporáneo veremos a continuación que es posible proponer un acercamiento entre descubrimiento y justifica-

6 Cfr. Popper, K., op. cit., pp. 30-32.

7 En sentido popperiano el término sería propiamente falsación. Pero optamos por dejar el término más genérico de contrastación, dado que el tema del artículo directamente no es el problema entre verificacionistas y falsacionistas. Con todo, sería más idóneo enmarcar este tema dentro del problema entre realistas y antirealistas.

8 Cfr. Nickles, Th., "Beyond Divorce: Current Status of the Discovery Debate", Philosophy of Science, Vol. 52, 1985, p. 180. 
ción, no tanto desde la oposición introducida por el problema del contexto, sino ante todo, desde la distinción entre lógica y razonamiento. Comenzaremos mediante un sucinto análisis de lo que propone tal distinción de contexto. Luego analizaremos críticamente la distinción entre una LIC y LDD a partir de la distinción por contextos para, finalmente, a modo de conclusión, sugerir que el divorcio -siguiendo la expresión de Nickles- entre descubrimiento y justificación es tan solo aparente.

\section{No todo descubrimiento implica una lógica del descubrimiento}

Uno de los aspectos nucleares para comprender la oposición entre una LIC y una LDD está en el problema de la demarcación, el cual permite establecer un criterio para discriminar adecuadamente un conocimiento científico de uno que no lo es, esto es, distinguir ciencia de pseudo-ciencia. Popper, recordando al propio Kant, señala: "[El conocimiento científico] no se interesa por cuestiones de hecho (el quid facti? de Kant), sino únicamente por cuestiones de justificación o validez (el quid juris? kantiano)"9.

En este sentido, no es casualidad que el problema de la demarcación se relacione con el problema de la inducción, esto es, "la cuestión sobre cómo establecer la verdad de los enunciados universales basados en la experiencia -como son las hipótesis y los sistemas teóricos de las ciencias empíricas". Cual el reverso a la demarcación, la inducción nos plantea el clásico problema de la justificación de los enunciados universales a los que la ciencia aspira.

Así, una proposición del tipo "todas las órbitas de los planetas describen trayectorias elípticas" no solo se articula dentro del complejo proceso de contrastación a la cual debemos someter dicho conocimiento, sino antes bien a la observación de casos concretos que la misma experiencia nos haya entregado. En otras palabras, la inducción elevada de la mera inferencia lógica a un método para justificar conocimiento implica un problema metodológico de difícil solución. Ello en vistas a que, recordando a Hume, la validez del conocimiento inferido dependerá de lo que ya sabemos, pues "no pueden existir argumentos demostrativos que prueben que casos de los que no hemos tenido experiencia son semejantes a aquellos en que sí la hemos tenido"10.

El caso histórico de Kepler con su De Motibus Stellae Martis ilustra este punto, ya que es a partir de la observación de la trayectoria no circular de la órbita de Marte que Kepler pudo inferir que todos los planetas podrían seguir el mismo patrón de comportamiento. Sin duda que el problema aquí no es la evidencia a posteriori que le ha dado la razón a Kepler $^{11}$, sino, metodológicamente hablando, cómo justificar un conocimiento que pende de un elemento que per se no es comprobable y, por ende, garantiza una universalidad y

9 POPPER, K., op. cit., pp. 27-28.

10 Hume, D., Tratado de la naturaleza humana, 4a edición, Tecnos, Madrid, 2008, p. 152. 
necesidad que no posee. A esto le llama Popper el problema de la inducción, entendida ésta como un dilema. Por una parte, la inducción como principio no puede ser demostrada deductivamente, ya que es contingente contrario a lo que establece toda demostración. Por otra parte, tampoco se puede apelar a la inducción ya que ello conllevaría a un principio de un principio en una regresión al infinito, imposibilitando la prueba misma ${ }^{12}$.

Sobre lo anterior, Popper propone, como es sabido, una lógica de la investigación basada en la falsación, esto es, en poder someter por vía negativa a contrastación las proposiciones que se postulan como conocimiento científico. Con ello se puede proponer una vía fiable para justificar un criterio de demarcación. Por lo mismo, las hipótesis no serían, en sentido estricto, objeto de interés para la ciencia, por cuanto sus génesis no pueden ser sometidas al proceso de falsación ${ }^{13}$. Este razonamiento establece en la base de la distinción señalada entre LDD y LIC una oposición entre hipótesis y contrastación, reformulando el método moderno entendido como una identidad entre descubrimiento y justificación. Si bien es importante la propia apreciación de Popper, respecto a que la ciencia comienza con problemas y termina con problemas, lo cierto es que el núcleo del programa poppereano está en un análisis lógico de la investigación científica y no, como se indicó, en articular una lógica de invención entendida como generación de hipótesis ${ }^{14}$. Esto significa que el método asociado a una LIC debe ser capaz "de contrastar críticamente las teorías y de escogerlas, teniendo en cuenta los resultados obtenidos en su contraste" $"$.

11 Tampoco el problema aquí es determinar cuáles fueron las razones que llevaron a Kepler a formular tal tipo de asociación entre un fenómeno particular y la hipótesis de sugerir que todo planeta debiera comportarse de manera análoga a Marte. Pese a ello, el caso de Kepler es un buen candidato para abordar el problema de una lógica del descubrimiento (Hanson, 1958).

12 Popper, K., op. cit., p. 28 y ss. Tampoco es plausible la probabilidad, “[...] pues si ha de asignarse cierto grado de probabilidad a los enunciados que se basan en inferencias inductivas, tal proceder tendrá que justificarse invocando un nuevo principio de inducción, modificado convenientemente [...]”.PopPER, K., ibid., p. 29. En rigor, lo que Popper sugiere es que la probabilidad, como la tesis de Reichenbach, modifican un aspecto formal, esto es, relativo a la frecuencia en que acontece un evento, pero metodológicamente sigue estando dentro de la misma deficiencia que la inducción. La alusión de Reichenbach a que negar la inducción es eliminar la ciencia, sería -interpretando a Popper en este punto-un quid pro quo.

13 La historia de la ciencia está llena de curiosas anécdotas al respecto. Pensemos, a modo de ejemplo, en un caso concreto. Kekulé y la figura hexagonal que representa la molécula de benceno. Kekulé, sin haber logrado hallar una solución a la fórmula que buscaba, al dormitar frente a una chimenea vio serpentear la figura para una molécula de benceno en la llamas de fuego, en la misma línea de la Einfühlung o intuición creadora. Cfr. Hempel, Carl, Filosofía de la Ciencia Natural, 6 edición, Alianza, Madrid, 1980, pp. 33-34.

14 En una conferencia del año 1963 Popper muestra el gran acercamiento que es posible establecer entre las ciencias naturales y las ciencias sociales. Al plantear dicha tesis Popper explica que la existencia de un problema es lo que orienta la actividad científica y que, a su juicio, se puede sistematizar en cuatro categorías: problemas, teorías, críticas y problemas. Pero, para Popper, el que la ciencia, tanto social como natural, comience con problemas y termine con problemas no implica que su interés metodológico está en la generación de problemas a modo de hipótesis. "De estas cuatro importantísimas categorías, la más característica de la ciencia es la de la eliminación de errores a través de la crítica". Cfr. Popper, K., "Modelos, Instrumentos y Verdad", en: Popper, K., El mito del marco común, Paidós, Barcelona-Buenos Aires, 1997, p. 157.

15 Popper, K., La lógica de la investigación científica, op. cit., p. 32. El filósofo austríaco distingue específicamente cuatro procedimientos para llevar a cabo dicha contrastación: 1) la comparación lógica de las conclusiones entre sí, 2) el estudio de la forma lógica de la teoría, 3) la comparación entre la teoría nueva y las ya existente de modo de sopesar si hay o no adelanto científico y 4) la contrastación en el orden de la aplicación empírica, es decir, qué conclusiones se pueden obtener de ella deductivamente. $C f r$., POPPER, K., Ibid. 
Conforme a este planteamiento, la filosofía de la ciencia adquiere un carácter normativo. Una teoría del método equivaldría a una cuestión de derecho, es decir, una LIC tiene que ver con el cómo ha de realizarse (de iure) la investigación en ciencia y no cómo tal o cual programa se ha realizado o realiza en la actualidad (de facto $)^{16}$. En síntesis, el descubrimiento como un método de generar una hipótesis y con ello plantear una teoría y una probable explicación no posee ningún tipo de peso epistémico, es decir, "ninguna información empleada al descubrir o construir un postulado puede ser justificado"17.

\section{a) Sentidos de descubrimiento}

Un punto importante para determinar críticamente la dicotomía entre LIC y LDD puede ser, paradójicamente, el propio título de la principal obra de Popper. Esta obra se publicó originalmente en alemán en 1934 bajo el título Logik der Forschung. En español la conocemos con el título de La lógica de la investigación científica. Como se expuso en los apartados anteriores, tal título -tanto en español como en alemán- obedece al proyecto poppereano de una teoría de la contrastación que no considera una lógica del descubrimiento. Mas, dicha obra se tradujo al inglés con el título de The Logic of Scientific Discovery. Tal como se ha hecho notar, el título introduciría una contradicción en el planteamiento de Popper, entre lo que la misma niega y lo que el título afirma: la posibilidad o imposibilidad de una LDD. En rigor el tema va más allá que una desafortunada traducción. Lo problemático es saber qué entendemos realmente por descubrimiento. En el fondo, "la bibliografía acerca del tema del descubrimiento científico es asombrosamente confusa por la ambigüedad y la complejidad del término descubrimiento"18.

Sobre lo anterior consideramos importante precisar dos posibles modos de entender descubrimiento. El primer modo de entenderlo sería: cuando una determinada teoría científica logra satisfactoriamente generar nuevo conocimiento ${ }^{19}$. El segundo modo de entenderlo se refiere a la generación y proceso de una teoría, independiente de su resultado. El primero es un sentido restringido, por cuando no todo descubrimiento es propiamente científico, al menos en el sentido poppereano, mientras que el segundo es un sentido amplio, pues considera el descubrimiento independiente de su "éxito epistémico" o "fuerza probatoria" ${ }^{20}$.

16 Nótese que, para la propuesta contemporánea predominante, tal distinción es válida incluso en los casos de programas de investigación científica que de facto posean fuerza probatoria.

17 NiCKLES, Th., op. cit., p. 183.

18 Aliseda, A., "Sobre la lógica del descubrimiento científico de Popper", Signos Filosóficos, Vol. VI, No 11, 2004, p. 118.

19 En el sentido poppereano implica satisfacer los 4 criterios expuestos en la nota 11.

20 Estos dos sentidos - restringido y amplio- tienen eco en la propuesta de Laudan. Sin embargo nuestra interpretación respecto a cómo entender restringido y amplio es opuesta a dicho planteamiento, ya que para Laudan lo restringido se refiere al descubrimiento entendido como la concepción de una idea, mientras que el sentido amplio se referiría a su juicio al proceso, esto es, desde la concepción de una idea hasta su justificación. Cfr. Laudan, L., "Why was the logic of discovery abandoned?" en: Nickles, Th., Scientific Discovery, Logic and Rationality, Reidel, pp. 173-183. La inversión que proponemos, como se podrá observar, no es casual, sino que obedece a la problemática oposición que se ha querido validar en filosofía de la ciencia entre justificación y descubrimiento a partir del tema del contexto. 
Pero la distinción tiende a ser débil, al menos en el sentido de oponer LIC y LDD cuando preguntamos lo siguiente: ¿hay un sentido para comprender lógica cuando distinguimos LIC de una LDD? Efectivamente una LDD no opera con los parámetros y categorías de una LIC, pero su contrario también es posible de plantear, es decir, tampoco una LIC opera con los parámetros y categorías de una LDD. El que un científico razone de un determinado modo para descubrir algo y que razone de otro modo para justificar algo no implica per se la existencia de una LDD y LIC respectivamente, del mismo modo que al presentar argumentos en una corte implique la existencia de una lógica de la ley ${ }^{21}$. Lógica y racionalidad no son sinónimos, aun cuando compartan un campo semántico afín. Esto último es importante de precisar al interior de la filosofía de la ciencia, ya que como es sabido, la lógica puede articular un argumento que no necesariamente implique una explicación concluyente para las ciencias experimentales. La lógica por sí sola no importa valor epistémico ${ }^{22}$. Gran parte del debate en torno a como entender esta aparente oposición se debe a atribuir una lógica a lo que sería propiamente un razonamiento. Esta problemática, conviene recordarlo, ya estaba presente en uno de los primeros tratados del método de la antigüedad, a saber, los Analíticos Posteriores de Aristóteles. Allí el filósofo griego aborda la cuestión entre demostración e intuición, anticipando la delgada línea que ha separado descubrimiento y justificación antes que complementarlos ${ }^{23}$.

\section{Conclusión}

Conforme a lo planteado en los apartados anteriores hemos establecido que la oposición entre una LDD y una LIC se basa en la distinción de los contextos, pero identificando una cuestionable sinonimia entre lógica y razonamiento. Con ello es posible volver a sugerir que no todo está dicho a favor de una LIC, concretamente respecto a la validez de abandonar el descubrimiento, esto es, al factor creativo como génesis de ideas nuevas en filosofía de la ciencia ${ }^{24}$.

Con ello no pretendemos sugerir un retorno avant la lettre al ideal moderno de mé-

21 Cfr. NickLes, Th., op. cit., p. 181.

22 NickLEs, Th., Idem.

23 Este tema excede los límites del presente artículo, pero resulta oportuno sugerir cómo ya en Aristóteles el problema, mutatis mutandi, estaba presente. Nos referimos a la propuesta aristotélica de la ciencia entendida como un saber demostrativo en oposición a un mero recordar, como acontece en Platón. La ciencia, como precisa Aristóteles, es saber en cierto sentido y en otro no, esto es, sí se sabe porque se puede establecer la causa, y no, en cuanto hay algo nuevo que nos permite descubrir el razonamiento y que antes no sabíamos, por ejemplo, que esta figura es un triángulo equilátero como aclara el propio filósofo. Cfr. ARIstóteles, "Analíticos Segundos", en Tratados de Lógica (Órganon), II, Gredos, Madrid, 1995, 71 a30-71 b5. Pero la misma demostración pende de principios primeros que no son demostrables y que conocemos mediante un conocimiento intuitivo (noético). Esto indica una afinidad clara en Aristóteles entre el conocimiento científico y las cosas, lo cual fue mayoritariamente abandonado por la discusión contemporánea en ciencia. Puede ser interesante recuperar el descubrimiento dentro del programa metafísico de Aristóteles, en cuanto que las hipótesis no son meras conjeturas como sugiere Popper, sino que en su razonamiento dicen algo de lo que son las cosas.

24 El sentido restringido en la interpretación de Laudan. 
todo. Proyectos emblemáticos como el de Leibniz o incluso el de Francis Bacon asumen una identificación entre mente y mundo que difícilmente hoy, incluso los partidarios de una LDD, estarían dispuestos a revivir al menos epistemológicamente hablando. Esto se debe a una restricción a dicha identificación entre mente y mundo a partir de la falibilidad promulgada por corrientes positivistas, lo cual introdujo un desplazamiento que va desde el diseño de teorías como búsqueda de un conocimiento verdadero a un conocimiento puramente conjetural.

Pero una concepción así entendida, como la resolución post hoc de problemas en un marco falibilista sugiere desde una LDD una crítica vital, vale decir, respecto de la inutilidad de una actividad científica sin un norte, como una búsqueda sin fin y que de alguna forma ha sido planteado por el denominado pesimismo inductivo ${ }^{25}$.

De aquí que la complementariedad entre descubrimiento y justificación no consiste en una lógica del método científico, sino en razones vitales. La ciencia es una actividad real del hombre y no un corpus de proposiciones que reside idealmente en un tercer mundo. En este sentido, es sugerente la reflexión de Artigas al respecto: “¿Qué sería una disciplina en la cual, siguiendo las ideas de Popper, se centrara la atención sólo o principalmente en las grandes novedades, buscando continuamente, por otra parte, contraejemplos que permitan detectar errores en ellas? Desde luego, la ciencia real no procede de ese modo" ${ }^{26}$. Revalorar el descubrimiento como una actividad creadora, en la misma línea que la actividad artística puede ser un posible punto de partida para una complementariedad entre descubrimiento y justificación cual el anverso y reverso de una misma realidad: “[...] la ciencia no es enemiga de las artes [...] ponerlas en oposición las mal interpreta y daña a ambas"27**

\section{Bibliografía}

Aliseda, Atocha, "Sobre la lógica del descubrimiento científico de Popper", Signos Filosóficos, Vol. VI, No 11, 2004, pp. 115-130.

25 El pesimismo inductivo, ya formulado primariamente en autores como Poincaré consiste en introducir una objeción escéptica: si la ciencia avanza a partir de la propia garantía metodológica que establece el falsacionismo, ¿qué garantía hay de proponer nuevas teorías si en el pasado muchas de ellas se mostraron posteriormente falsas? Si bien el pesimismo inductivo ha sido ampliamente rebatido, nos servimos de su planteamiento para efectos de ilustrar con mayor claridad nuestro análisis. Para discusiones actuales de las objeciones al pesimismo inductivo, $c f r$. PARK, Seungbae, "A Confutation of the Pessimistic Induction", Journal for General Philosophy of Science, Volume 42, 2010, pp. 75-84; Mizrahi, Moti, "The Pessimistic Induction: A Bad Argument gone too far", Synthese, Volume 190, 2013, pp. 3209-3226.

26 Artigas, M., Filosofía de la ciencia experimental, $2^{\text {a }}$ edición, Eunsa, Pamplona, 1992, p. 101.

27 Goodman, N., Of Mind and Other Matters, Harvard University Press, Cambridge, Massachussets- London, 1984, p. 5.

*Artículo recibido: 5 de noviembre de 2014. Aceptado: 11 de diciembre de 2014. 
JAVIER KASAHARA B.

Aristóteles, “Analíticos Segundos”, en Tratados de Lógica (Órganon), II, Gredos, Madrid, 1995.

Artigas, Mariano, Filosofía de la ciencia experimental, $2^{\text {a }}$ edición, Eunsa, Pamplona, 1992.

Hempel, Carl, Filosofía de la Ciencia Natural, 6a edición, Alianza, Madrid, 1980.

Hume, David, Tratado de la naturaleza humana, 4ª edición, Tecnos, Madrid, 2008.

Goodman, Nelson, Of Mind and other matters, Harvard University Press, Cambridge, Massachussets- London, 1984.

Kordig, C. R., "Discovery and Justification”, Philosophy of Science, Vol. 45, 1978, pp. 110-117.

Laudan, L., "Why was the logic of discovery abandoned?" en: Nickles, Thomas, Scientific Discovery, Logic and Rationality, Reidel, pp. 173-183.

Mizrahi, Moti, “The Pessimistic Induction: A Bad Argument gone too far", Synthese, Vol. 190, 2013, pp. 3209-3226.

Nickles, Thomas, "Beyond Divorce: Current Status of the Discovery Debate", Philosophy of Science, Vol. 52, 1985, pp. 177-206.

Park, Seungbae, "A Confutation of the Pessimistic Induction", Journal for General Philosophy of Science, Volume 42, 2010, pp. 75-84.

Popper, Karl, El mito del marco común, Paidós, Barcelona-Buenos Aires, 1997. -, La lógica de la investigación científica, Tecnos, Madrid, 1980. 